\title{
ACUTE VISUAL FAILURE AS A NEUROSURGICAL EMERGENCY*
}

\author{
BY
}

\author{
ALFRED UIHLEIN \\ Section of Neurological Surgery, Mayo Clinic and Mayo Foundation, $\uparrow$ Rochester, Minnesota
}

MORE often than not visual failure is the result of a disorder of the globe, and hence lies within the province of the ophthalmologist. However, a lesion involving the optic pathways may occasionally be responsible for acute loss of vision (List, Williams, and Balyeat, 1952; Love, Dodge, and Bair, 1955; Uihlein, Balfour, and Donovan, 1957) and at times such a lesion is overlooked because a thorough medical examination was not conducted when the patient first consulted his physician (Baker and Rucker, 1950; Jefferson, 1955; MacCarty, Lillie, Daly, Hollenhorst, and Holman, 1957). Ideally, if irreparable visual loss is to be prevented, the cause should be determined early enough to allow effective treatment. This requires a thorough medical examination, which should include ophthalmological investigation, roentgenograms of the skull, neurological examination, and accurate plotting of the visual fields (Hughes, 1954). In the more difficult diagnostic problems, laminagrams of the skull and cerebral angiography, as well as sagittal sinus venography and pneumo-encephalography, may be required if the condition of the patient will tolerate these procedures (Norlén, 1952).

A review of the records of 310 patients who came to the Mayo Clinic because of unilateral loss of vision was conducted by Uihlein and Weyand (1953). Lesions of structures of the eyeball and glaucoma, in that order, caused impaired vision in 154 (49.7 per cent.) of the patients. Lesions involving the retrobulbar portion of the optic nerve were noted in 108 (34.8 per cent.) of the 310 patients, and additional analysis of these 108 cases showed that in 17.6 per cent. vision was lost because of a pituitary tumour, in 13.9 per cent. because of a meningioma, and in 14.8 per cent. because of an unverified chiasmal lesion. The combined diagnoses of craniopharyngioma, aneurysm, or tumour were made in only thirteen $(12.1$ per cent.) of the 108 cases. On the basis of these findings it is apparent that the possibility of the presence of a neurosurgical lesion must always be considered in the differential diagnosis of acute visual failure.

On the basis of a review of our patients who have consulted us because of

* Read at the combined meeting of the Society of British Neurological Surgeons and the 36th Annual Meeting of the Irish Ophthalmological Society in Dublin, May 16-18, 1957.

$\dagger$ The Mayo Foundation, Rochester, Minnesota, is a part of the Graduate School of the University of Minnesota. 157 
this type of visual failure, we have compiled a Table which we feel represents the most common clinical entities that may produce a sudden decrease in visual acuity. An attempt has been made to list these rare conditions in the order of frequency. Most, but not all, of these conditions will require neurosurgical investigation.

\title{
TABLE
}

\section{COMMON CAUSES OF DECREASED VISION IN ORDER OF FREQUENCY}

\author{
1. Pituitary tumour \\ 2. Tumour of optic nerve or chiasma \\ 3. Supraclinoid aneurysms \\ 4. Parasellar lesions \\ 5. Carotid arterial thrombosis \\ 6. Third-ventricle hydrocephalus \\ 7. Chiasmal arachnoiditis \\ 8. Fracture of anterior cranial fossa \\ 9. Basofrontal tumour of skull \\ 10. Pseudo-tumour cerebri
}

It is not my intention to review the diagnostic features in all these conditions, but salient clinical findings in some of them are outlined. The case reports illustrate the diagnostic points that indicated the need for early neurosurgical intervention.

\section{Case Reports}

Case 1, a lawyer aged 64, had apparently been well until 8 days before he was admitted to a Rochester hospital. Apart from an incompletely treated syphilitic infection contracted in his early twenties, he had apparently always been in good health.

Six days before admission he began to vomit, 2 days later he noted loss of visual acuity in the left eye, and the next day his left eyelid began to droop. Lumbar puncture and examination of the cerebrospinal fluid (performed at home) revealed increased pressure, with 80 to 90 erythrocytes per c. $\mathrm{mm}$.

On admission he said he was blind in the left eye and that he noted "spotty" blindness of the right eye. He complained chiefly of generalized malaise and headache, with nausea. He had noted a decrease in his sense of smell for about a year.

The patient was tall, with acromegaloid facial features, but the hands and feet were normal. He was drowsy but oriented. Blood pressure 132/80. Pulse rate 68. Except for a bilateral Babinski response, apathy, and Grade 3 palsy (on the basis of 1 to 4) of the third cranial nerve on the left, the results of the neurologic examination were negative. The left pupil was dilated and fixed, and the lid drooped slightly, but perception of light was present in both eyes.

At ophthalmological examination the visual acuity in the left eye was counting fingers and $14 / 42(+2.06)$ in the right. The visual field of the right eye was contracted temporally below. There was no papilloedema.

A roentgenogram of the skull showed enlargement of the sella turcica, with decalcification (Fig. 1, opposite). The pineal calcification was displaced slightly to the left. Results of laboratory procedures, except the results of serologic tests, were normal. The basal metabolic rate was -23 .

Two days after he had been admitted to the hospital the patient's condition became worse. Temporal anopsia and a central scotoma on the right developed, and the left eye was blind (Fig. 2, opposite). 


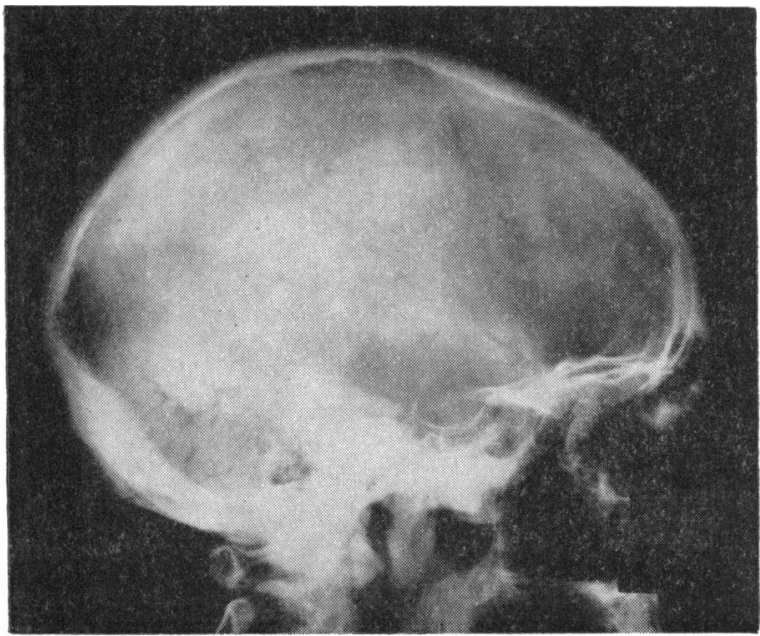

Fig. 1.-Lateral roentgenogram of skull in Case 1.
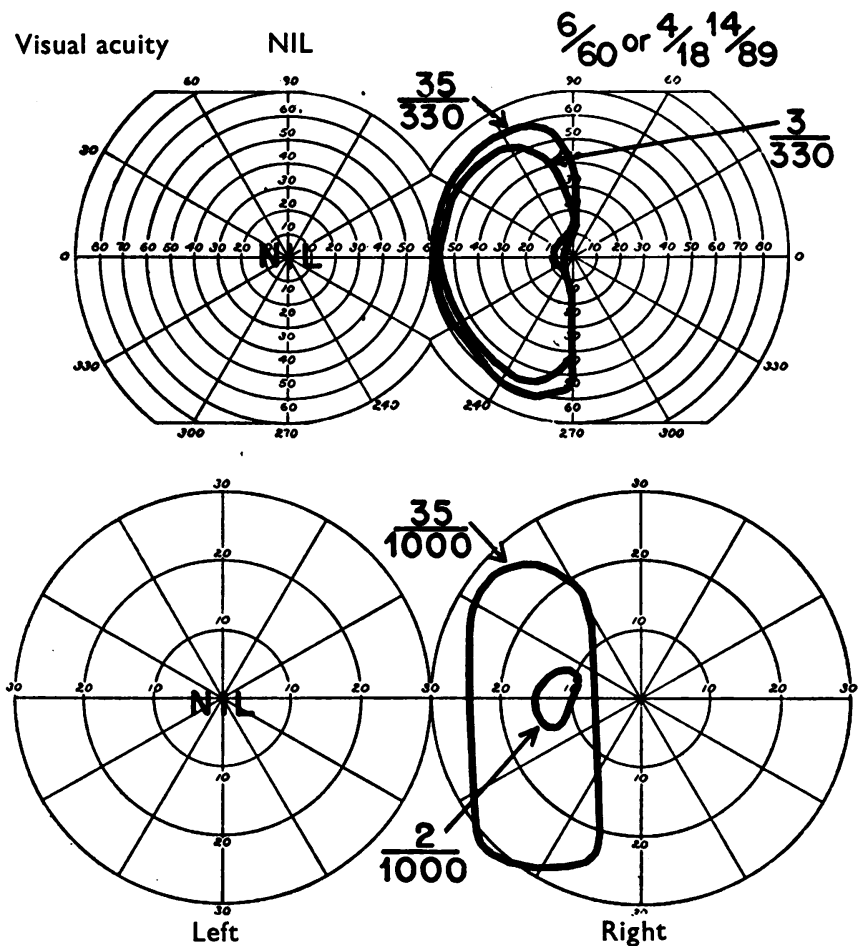

Fig. 2.-Visual fields as plotted on initial examination in Case 1.

Carotid angiograms (Fig. $3 a, b$, overleaf), made with the aid of cross-filling from collateral circulation and with thorium dioxide (thorotrast), excluded an aneurysm of the circle of Willis, but the carotid siphon was deformed as by the pressure of a prechiasmal lesion.

An emergency craniotomy was performed on the left on August 21, 1954. Bloody cerebrospinal fluid was found in the chiasmal cistern. The optic nerves were bowed upward and back by a dark-coloured mass. The left optic nerve was ecchymotic. After 
a spinal-puncture needle had been inserted into this mass to exclude the presence of an aneurysm, the capsule of the mass was incised. Much black blood was evacuated in this

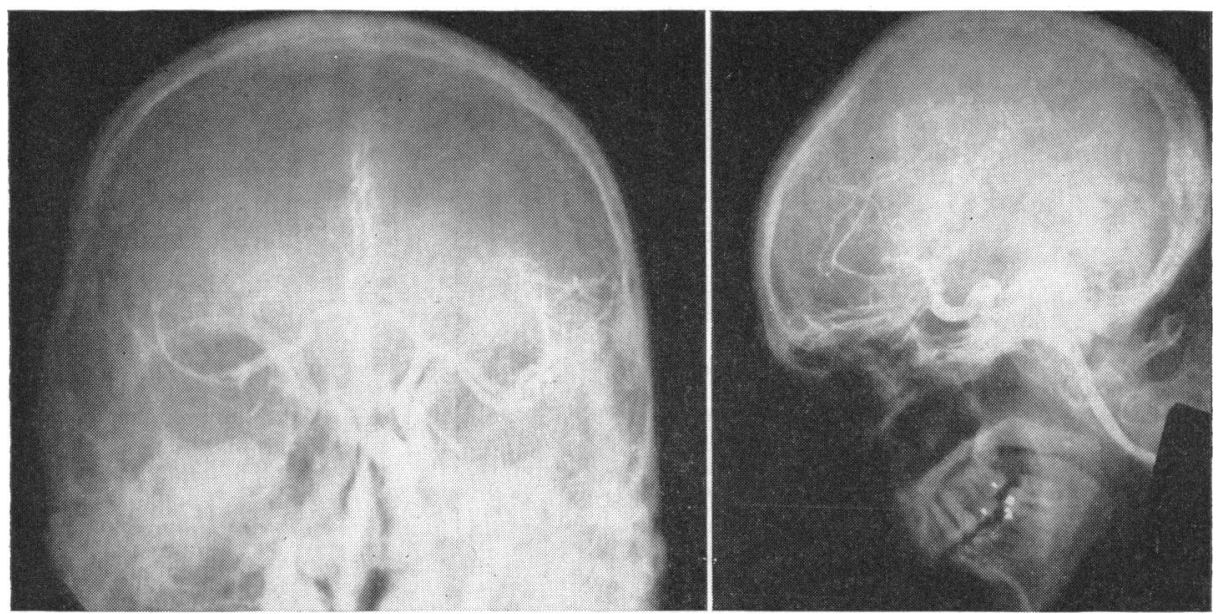

FIG. 3(a, b).-Antero-posterior and lateral carotid angiograms illustrating elevation of both anterior cerebral arteries in Case 1.

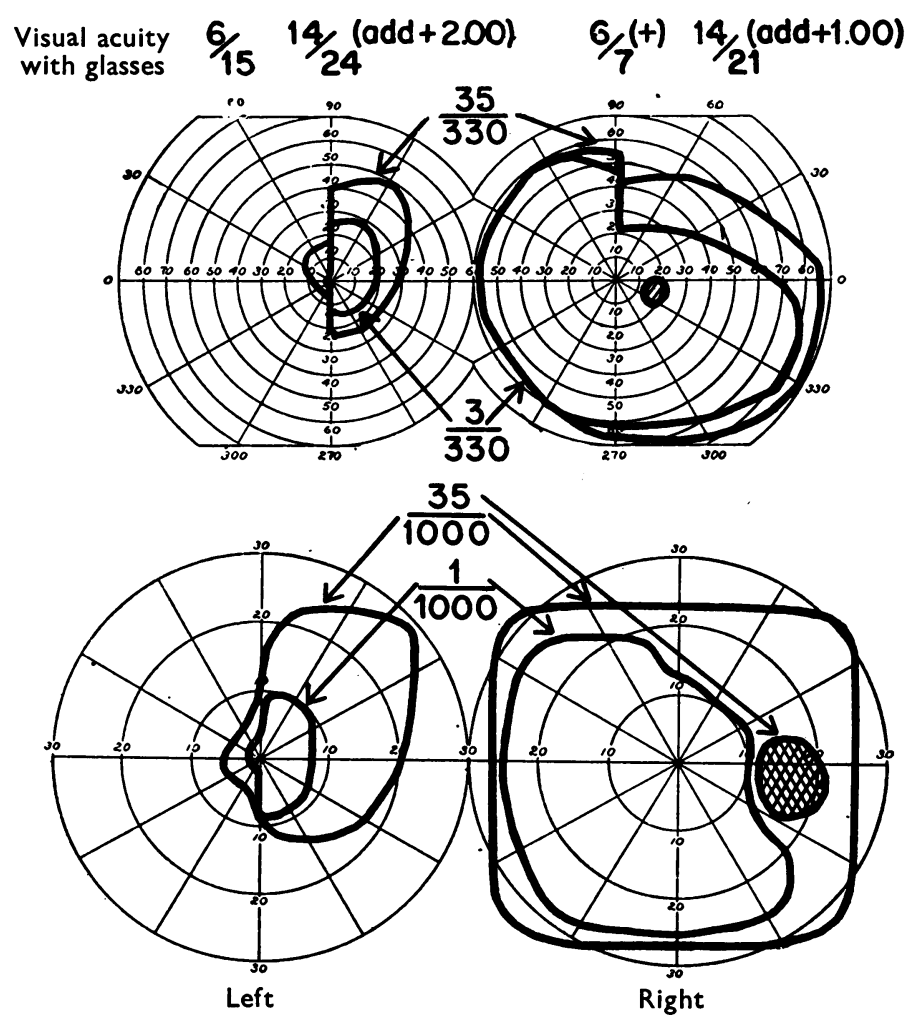

FIG. 4.-Visual fields plotted 9 days after operation in Case 1. 
way, and adenomatous tissue of the pituitary gland plus the capsule were removed. All pressure was removed from the optic pathways. The pathologist reported the lesion to be an infarcted haemorrhagic adenoma of the pituitary body.

Convalescence was uneventful. Perception of light on the left was noted by the patient at the end of the second post-operative day, and the third-nerve palsy was lessening. Nine days after the operation marked improvement in central vision in the left eye was evident, with a filling-in of most of the right temporal field and much of the nasal field of the left eye (Fig. 4).

A re-examination on December 6, 1954, disclosed Grade 1 pallor of the left optic disc. The right optic disc was normal, the right visual field was almost normal, and the left was filling out (Fig. 5). Visual acuity was 6/12 in the left eye and 6/10 in the right. Reexamination of visual fields on August 10, 1955, showed no change since the time of the last examination.

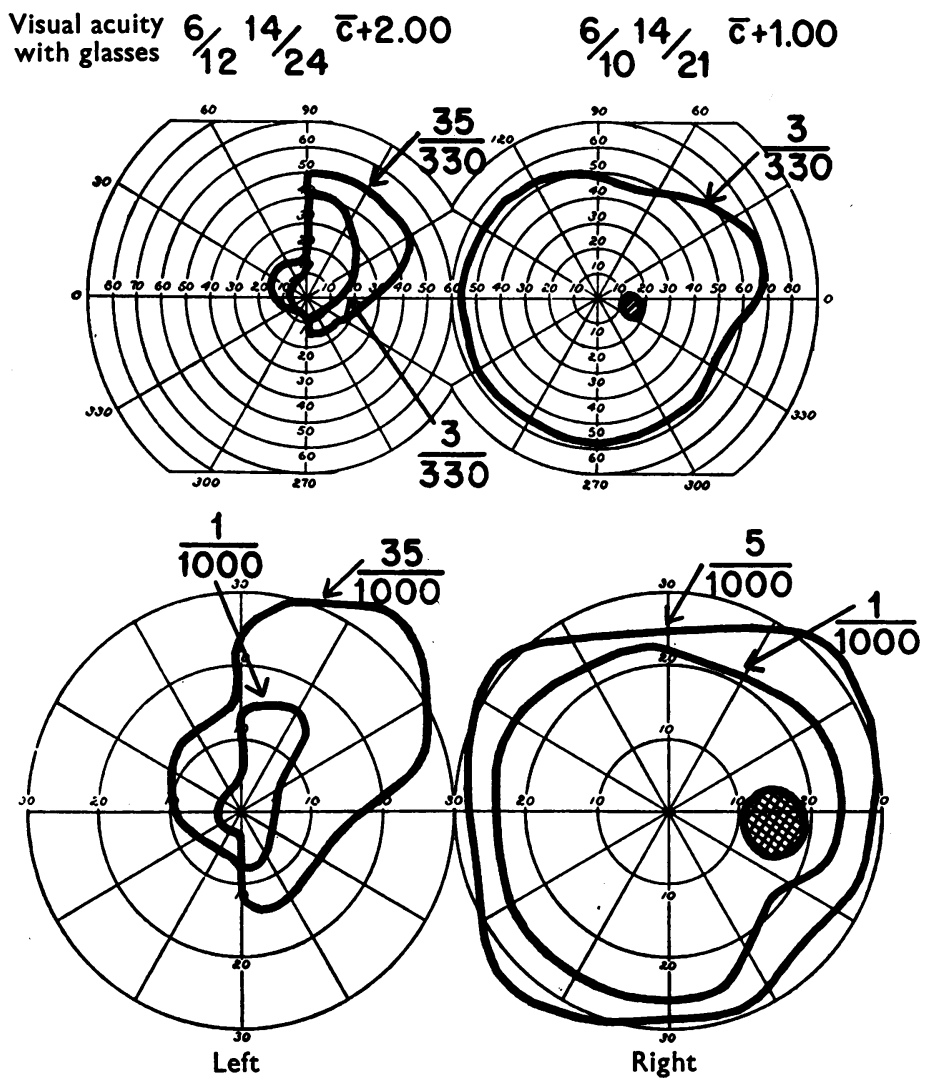

Fig. 5.-Visual fields plotted 4 months after operation in Case 1.

The condition of this patient represents an acute haemorrhage into an unsuspected adenoma of the pituitary body. The vision of this patient was saved by early diagnosis, which permitted definitive surgical treatment for removal of the cause. 
Case 2, a boy aged 12, was referred to the clinic on August 24, 1954. The referring physician had diagnosed retrobulbar neuritis; 2 weeks earlier, the patient had noted blurring of vision on the left while target shooting, and 10 days later he could not see objects with the left eye when the right eye was closed, except in the temporal field. On arrival at the clinic, he complained of a throbbing and dull headache, with little or no vision in the left eye.

Results of the physical examination were essentially negative. Roentgenograms of the head and optic canal disclosed no abnormality.

Visual acuity was hand movements at 1 foot with the left eye, and 6/6 and 14/21 without correction in the right. The extra-ocular movements were normal. The ocular tension was $20 \mathrm{~mm}$. Hg each eye. The visual field of the right eye was full, but was confined to the upper quadrants in the left. Both optic nerve heads appeared normal. At this stage retrobulbar neuritis appeared to be the most likely diagnosis.

Two days later the patient complained that vision in the right eye had become blurred; nausea and vomiting began that morning, and lethargy developed. On August 27, 1954, the left eye was blind, and only a residual nasal field remained in the right eye (Fig. 6). The pupils were dilated and equal; the left responded poorly to light.
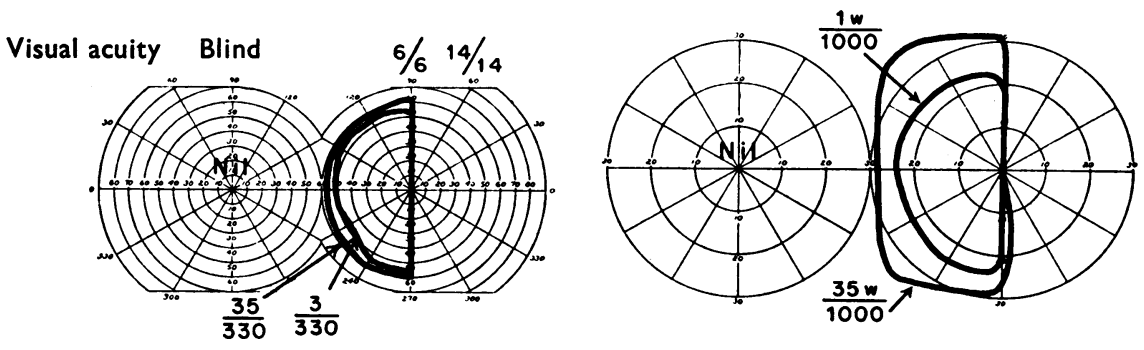

Further loss of visual field. Residual nasal field in right eye. Left eye is blind.

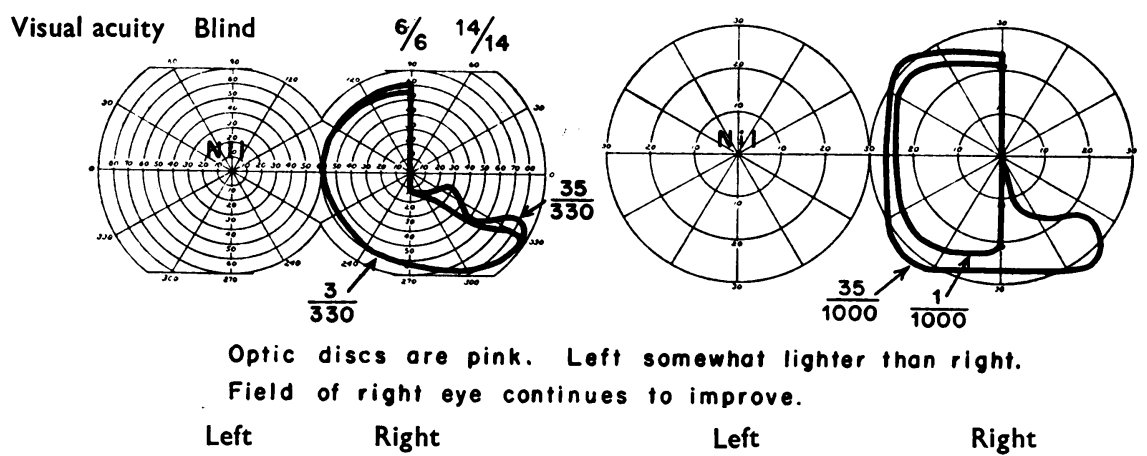

FIG. 6.-Visual fields plotted on August 27, 1954, in Case 2.

An emergency craniotomy performed on the same day disclosed a haemangio-endothelioma of the left optic nerve and left half of the chiasma, with intramedullary rupture of the optic nerve. Acute haemorrhage had occurred within the haemangio-endothelioma (Fig. 7), compressing the anterior portion of the third ventricle. The segment of involved nerve was resected from the globe up to and including that portion of the chiasma on the left. Post-operative $x$-ray therapy was administered. 


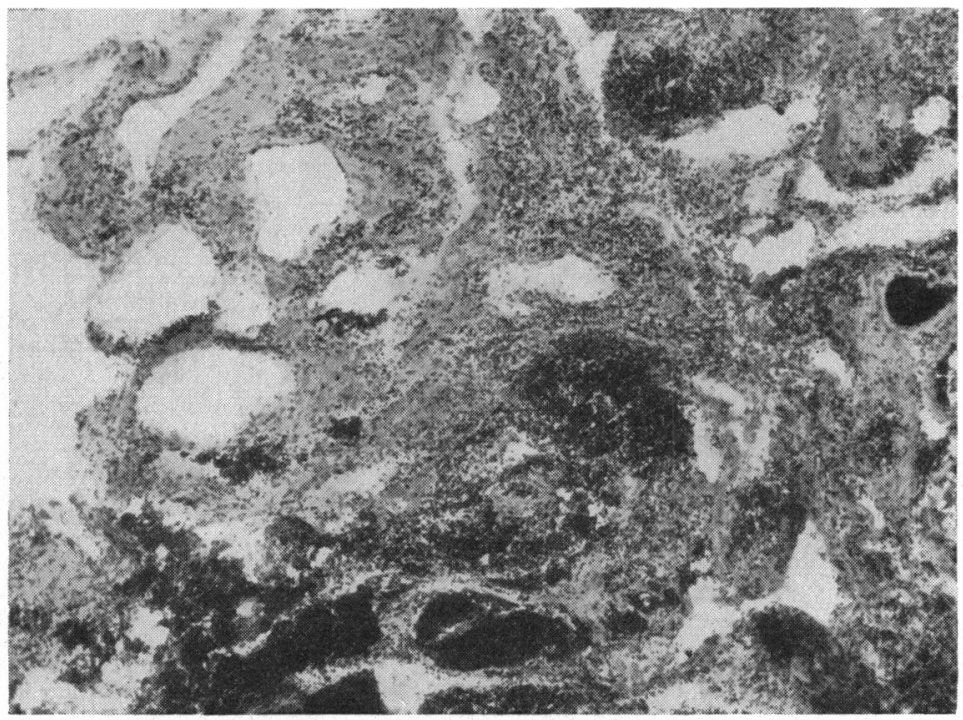

Fig. 7.-Section of pathological specimen in Case 2. Haematoxylin and eosin $\times 75$.

Twelve days after the operation the right visual field had recovered the inferior temporal isle (Fig. 8).
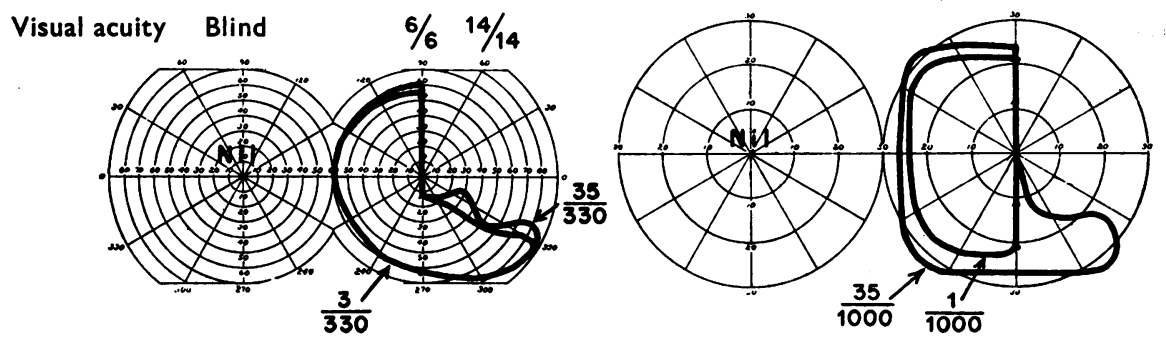

Fig. 8.-Lower visual fields plotted on September 8, 1954, after operation in Case 2.

Four months later there was Grade 1 pallor of the right optic disc, and Grade 3 pallor of the left optic disc (on the basis of 1 to 4). There was no additional loss of vision in the right eye, and the return of the temporal field was of the type that may follow resection of a portion of the left half of the chiasma (Fig. 9a).
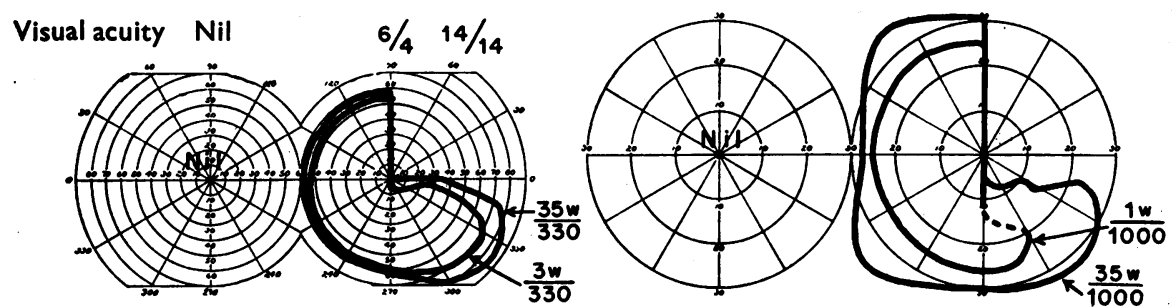

FIG. 9(a).-Visual fields plotted 4 months after operation in Case 2. 
The visual fields were essentially unchanged when the patient was last examined approximately a year later (Fig. $9 b$ ).

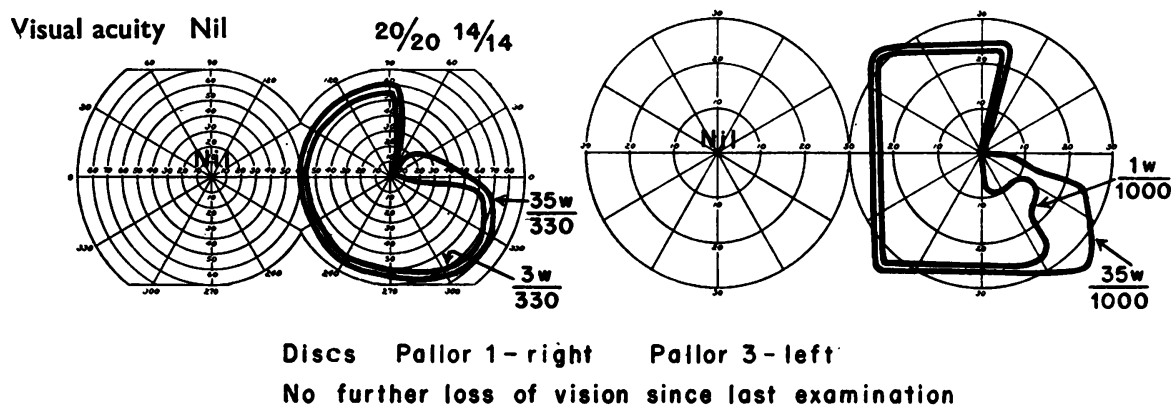

Fig. 9(b).-Visual fields plotted approximately 1 year after operation in Case 2 (July 12, 1955).

Case 3, a boy aged 10, was admitted to the hospital on November 6, 1952, with a 5 weeks' history of headaches and progressive loss of vision in the right eye. The boy had been in good health until 5 weeks previously, when his head had been banged against a wall by playmates.

On November 1, 1952, he was suffering from headache and the visual acuity was perception of light in the right eye. The pupillary reflex was down on the right. The visual fields on gross testing suggested upper temporal quadrant anopsia in the left eye. On the next day the anopsia had changed to left temporal hemianopia. The Babinski reflex was elicited on the right. The neck was stiff and the rectal temperature was $102^{\circ} \mathrm{F}$. The peripheral leucocyte count was 12,600 per c. $\mathrm{mm}$. blood. These observations were made by a physician in the patient's home town.

The cerebrospinal fluid pressure was $114 \mathrm{~mm} . \mathrm{Hg}$, and the total protein content was $55 \mathrm{mg} . / 100 \mathrm{ml}$.

On November 3, ventriculography (carried out in the patient's home town) suggested inadequate filling of the chiasmal cistern, without shift of the ventricular system.

On November 7 (at the Mayo Clinic), an ophthalmological examination, incomplete because of the acute illness, revealed blindness in the right eye and complete temporal anopsia in the left. Roentgenograms of the head revealed no decalcification of the sella turcica, and views of the optic canals seemed to show that they were normal. Immediate craniotomy disclosed a ballooned-out right optic nerve which at first glance resembled an aneurysm. A needle was inserted in the mass and dark blood was obtained. The nerve had been disrupted by a clot. The operation was terminated at this stage, as the pathologist could not identify tumour tissue in frozen sections. On the seventh post-operative day (November 14), however, the patient became lethargic and experienced Jacksonian seizures of the left arm and left side of the face. Re-exploration at this time revealed bloody cerebrospinal fluid trapped subdurally beneath the frontal lobe. This fluid was released and the optic chiasma was re-explored. The right optic nerve appeared macerated. Biopsy revealed a Grade 1 telangiectatic astrocytoma (Fig. 10, opposite). The optic nerve was divided distal to the mass at the optic foramen, which appeared enlarged, and the right nerve and right half of the chiasma were resected.

On November 21, 1952, visual field of the left eye had improved (Fig. 11a, opposite). On December 18, 1953 (Fig. 11b, opposite), the visual acuity in the left eye was normal with no additional loss of its remaining field. Examinations in 1954 disclosed no change.

The sequence in this case was one of a progressive chiasmal lesion, beginning prechiasmally on the right and extending to involve the chiasma. Trauma to the head may have produced the haemorrhagic disruption of the nerve and the rapid visual loss. 


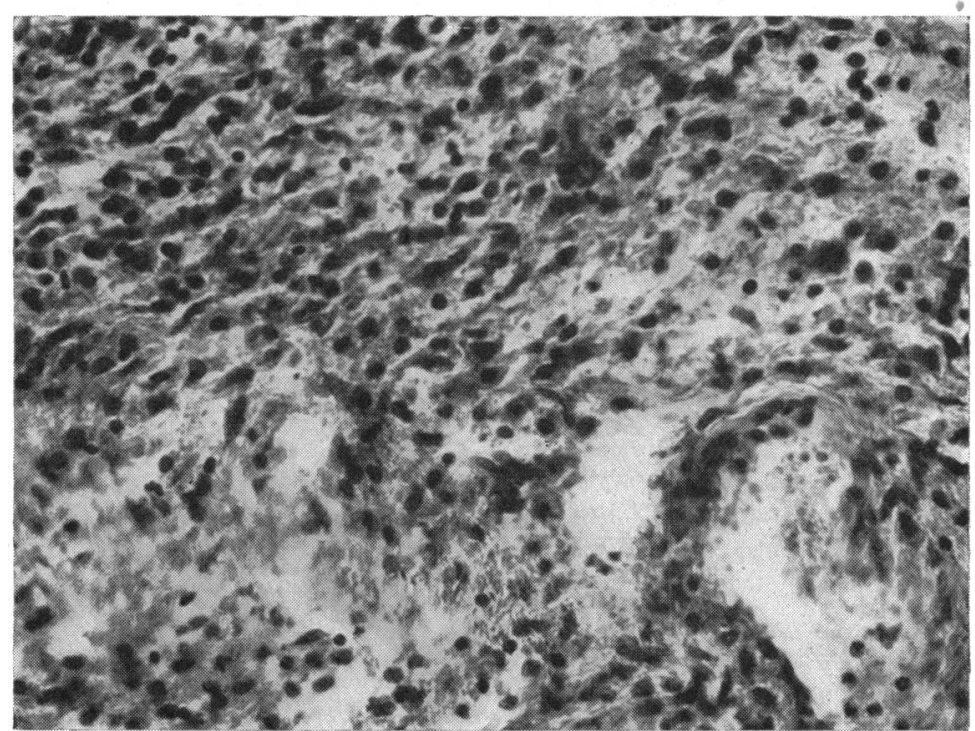

Fig. 10.-Section of pathological lesion in Case 3. Haematoxylin and eosin $\times 300$.

Visual acuity
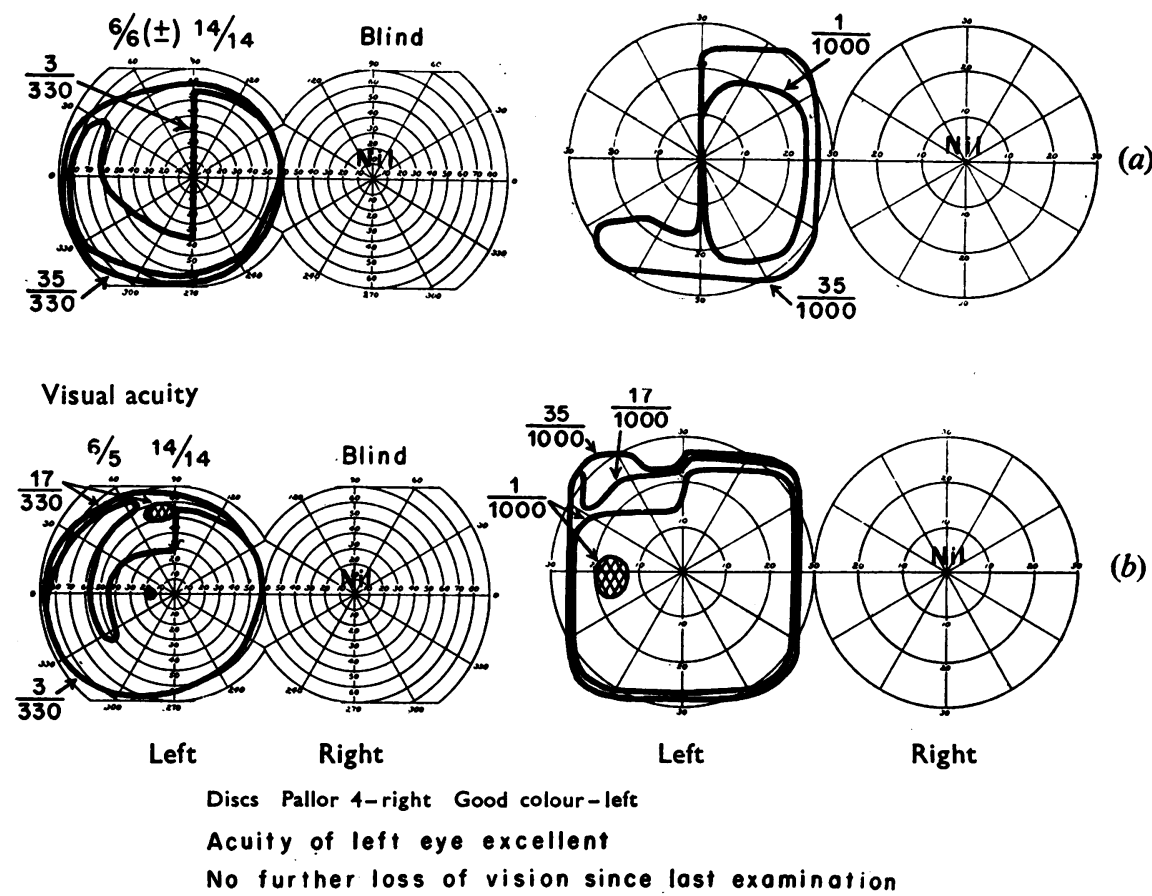

(b)

FIG. 11(a).-Visual fields in Case 3 plotted on November 21, 1952.

(b). - Visual fields in Case 3 plotted approximately 1 year later (December 18, 1953). 
Case 4, a woman aged 52, was referred to the clinic with a diagnosis of multiple sclerosis on January 2,1957. She had noted blurring of vision in the temporal field of the right eye in the summer of 1953, but this impairment of vision had not progressed.

In May, 1956, she had begun to notice blurring of vision in the left eye, and the impairment was progressive until the time of her admission to the clinic. She had been unable to read since November, 1956. Two pneumo-encephalograms, made in the patient's home city, were reported as disclosing nothing abnormal. The content of protein in the cerebrospinal fluid was $107 \mathrm{mg} . / 100 \mathrm{ml}$.

The results of a general medical examination at the clinic were essentially negative, except for the presence of obesity and hypertension, the blood pressure being 180/130. The patient said she had not had headaches, polyuria, or loss of pubic or axillary hair, or other signs of pituitary dysfunction.

The results of neurological examination were completely negative, except in respect to the visual pathways. On January 3 the visual acuity was 20/70 in the left eye and 20/200 in the right. Grade 2 pallor of the right optic disc and Grade 1 pallor of the left optic disc were noted. The arterioles were narrowed to Grade 1, with minimal sclerosis and focal constriction. The visual fields (Fig. 12) showed bitemporal hemianopia, such as may develop in the presence of a chiasmal lesion. The ophthalmologist could not believe that this was a manifestation of multiple sclerosis.

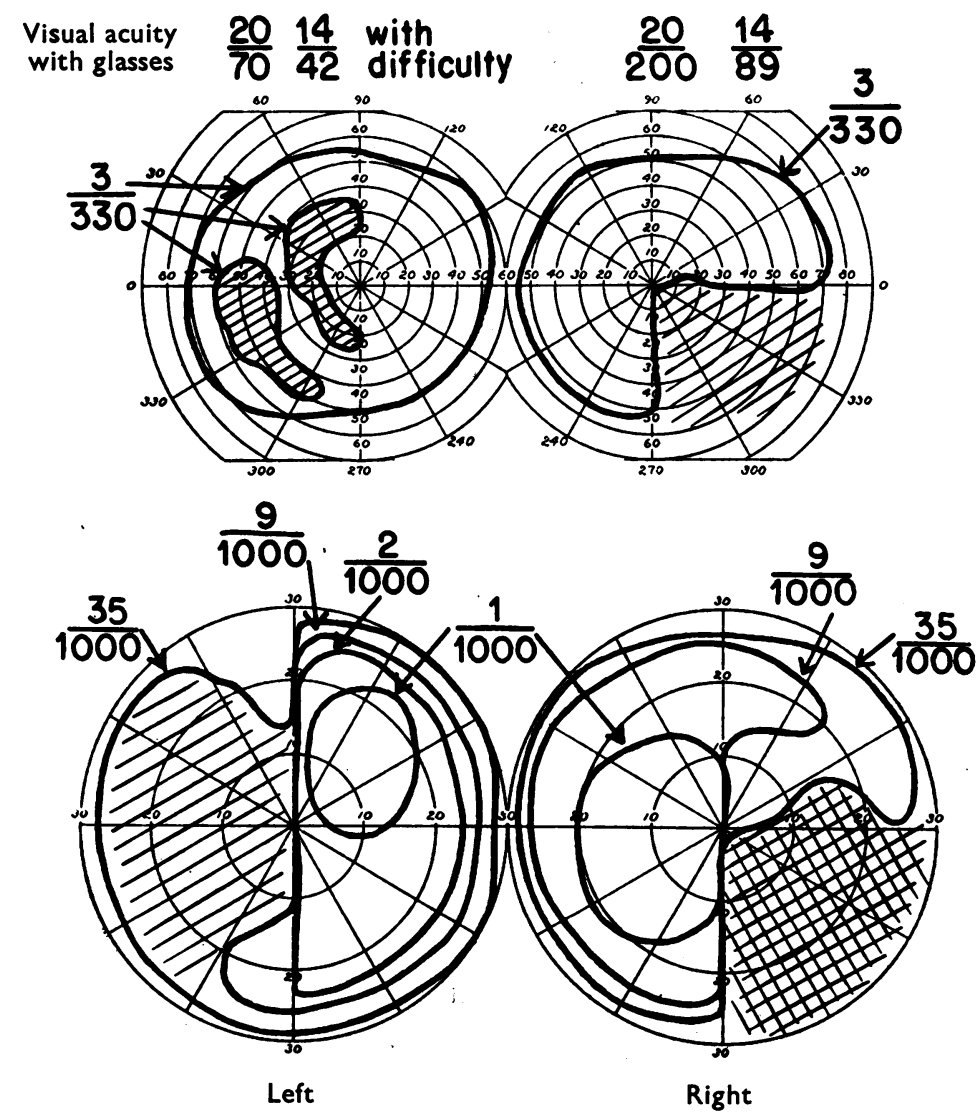

FIG. 12.-Visual fields plotted on January 3, 1957, in Case 4. 
On January 7, bilateral carotid angiography was carried out. This demonstrated a large aneurysm of the internal carotid artery beneath the clinoid process (Fig. 13a,b); there was cross-filling from left to right, but the aneurysm did not fill from the left. It appeared to be bilobate. The interpretation was that the aneurysm might be divided by the chiasma or the optic nerve on the right.

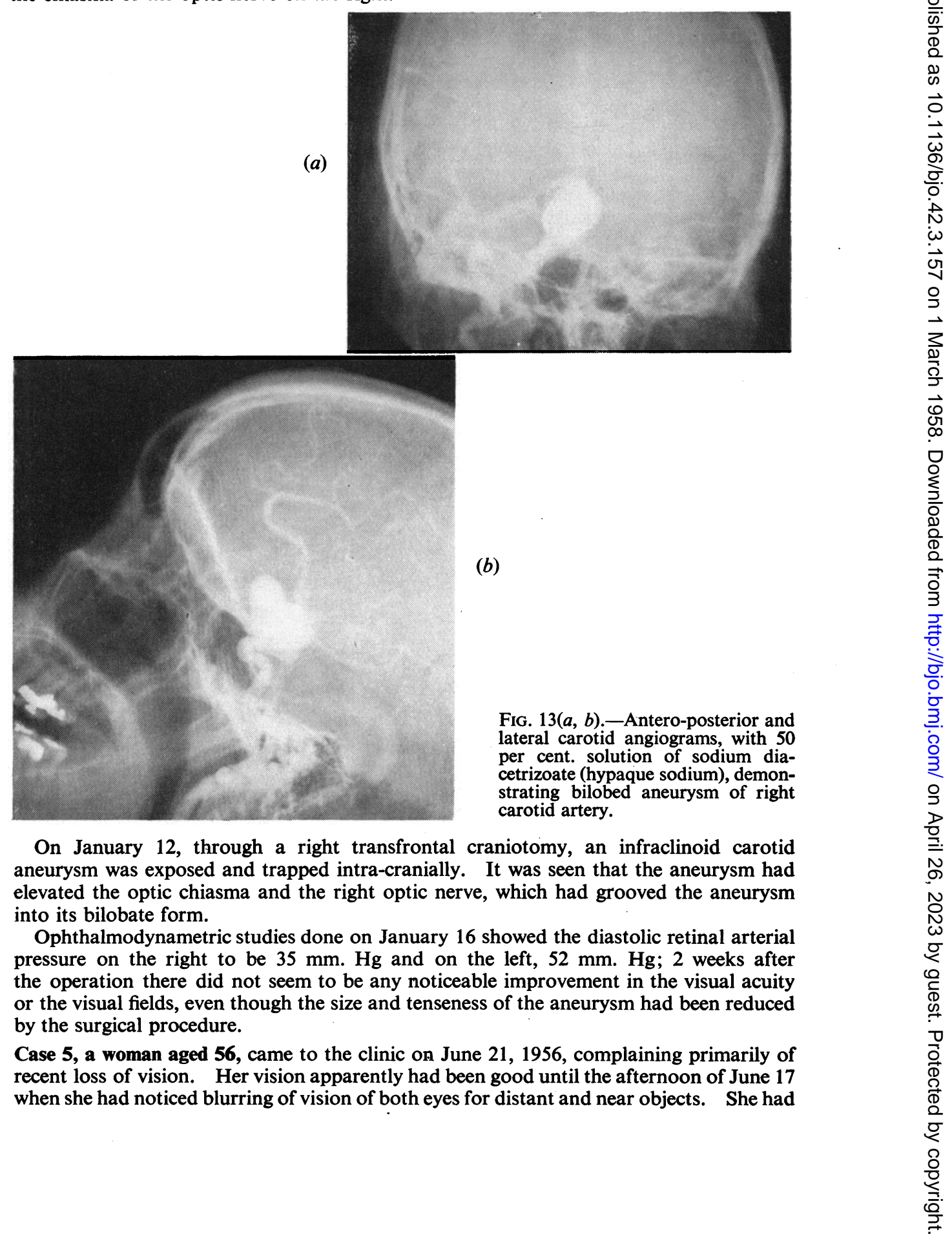


been able to read the newspaper that night, but by the next day she could see only the headlines. She had not taken any drugs. There was no history of the use of alcohol or tobacco, or of headaches or other symptoms. Splenectomy had been done in 1953 to relieve secondary thrombocytopenic purpura. The patient recalled that she had used reading glasses for more and more tasks in the previous years, although she had worn glasses intermittently for about 8 years. Physical examination and laboratory tests gave normal results. Roentgenograms of the head disclosed a normal-looking sella turcica and no evidence of erosion of the clinoid processes. The results of the neurological examination, except in respect to vision, were normal. The patient was alert and responded well.

With the right eye she could count fingers at 3 feet, but with difficulty; in the left eye the visual acuity was $20 / 400$. Pupillary reflexes, extra-ocular movements, and peripheral fields appeared to be normal. On June 22 (Fig. 14) the optic discs were found to be of good colour with a rather extensive scotomatous bitemporal lesion due to involvement of the optic chiasma and both optic nerves. The sudden onset and gradual progression in 5 days suggested an expanding lesion, possibly haemorrhage into a pituitary adenoma, an aneurysm, or other prechiasmal lesion. Optic neuritis was considered, but the progressive visual loss seemed to be against this.

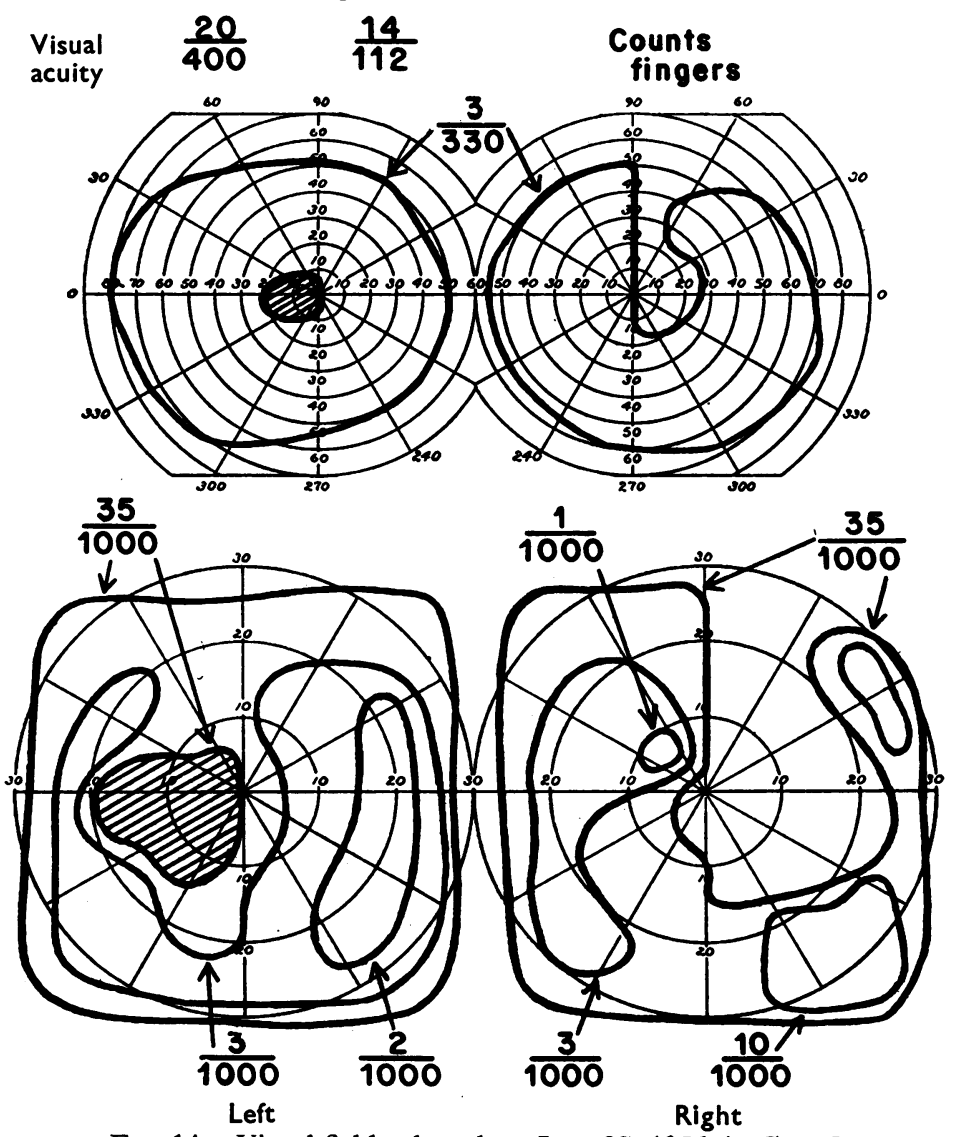

Fig. 14.-Visual fields plotted on June 22, 1956, in Case 5.

The patient was admitted to hospital, and the next morning said she could not see as well as on the night before. Bilateral carotid angiography on June 23 did not reveal any 
displacement of the carotid siphon. Because of progressive visual loss, emergency craniotomy seemed indicated. Just before lapsing into unconsciousness for the craniotomy, the patient said she was blind.

A right transfrontal craniotomy revealed no evidence of tumour apart from a mild arachnoiditis about the chiasma and optic nerves. The ophthalmic arteries seemed to be more prominent than usual, and appeared to bow the optic nerves upward. There was no evidence of an aneurysm. Six hours after craniotomy, the patient reported that she could see light with each eye. The pupils were active and equal, and the next day the patient said she could see her own hand as a shadow.

On July 3 (Fig. 15), the visual fields demonstrated a remarkable recovery from the complete blindness of 10 days before. Defects were of the chiasmal type, and at this time the patient could have been recovering from chiasmal neuritis.
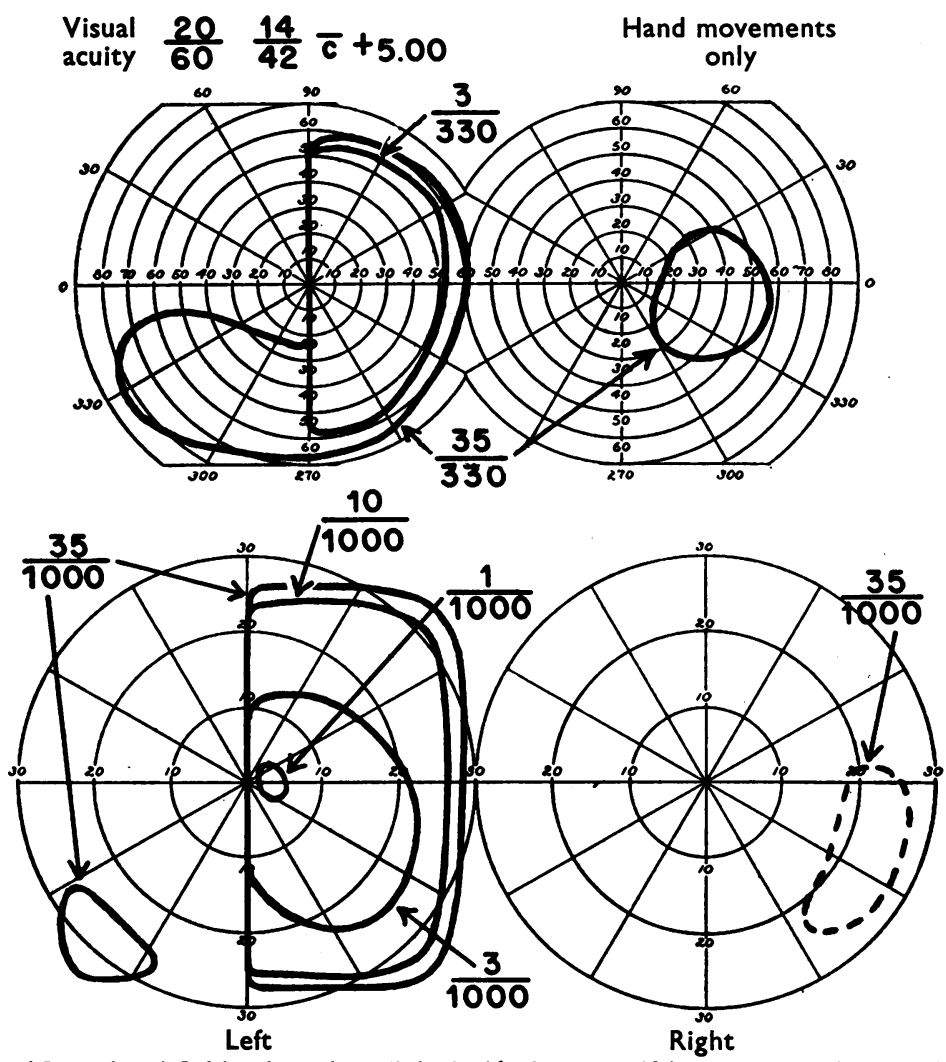

Fig. 15.-Visual fields plotted on July 3, 1956, on twelfth post-operative day in Case 5.

By July 26 the ocular fundi were normal, and the visual fields had almost returned to normal (Fig. 16, overleaf). There was 20/20 vision in both eyes.

The patient did well until about $3 \frac{1}{2}$ months later, when vision in the left eye began to fail again. Vision in that eye failed progressively until she presented herself again for examination on November 17, 1956. It was found that the right eye was again blind. Nicotinic acid was prescribed, and the patient again recovered vision gradually. Central scotomata were present in both fields of vision on December 15, 1956 (Fig. 17, overleaf).

On December 22, 1956, it was found that vision in the left eye had continued to improve, though that in the right eye was very poor. On January 5, 1957 (Fig. 18, overleaf), 
Grade 1 pallor of both optic discs was seen. The visual field of the left eye continued to improve; the right remained about the same or possibly somewhat worse.
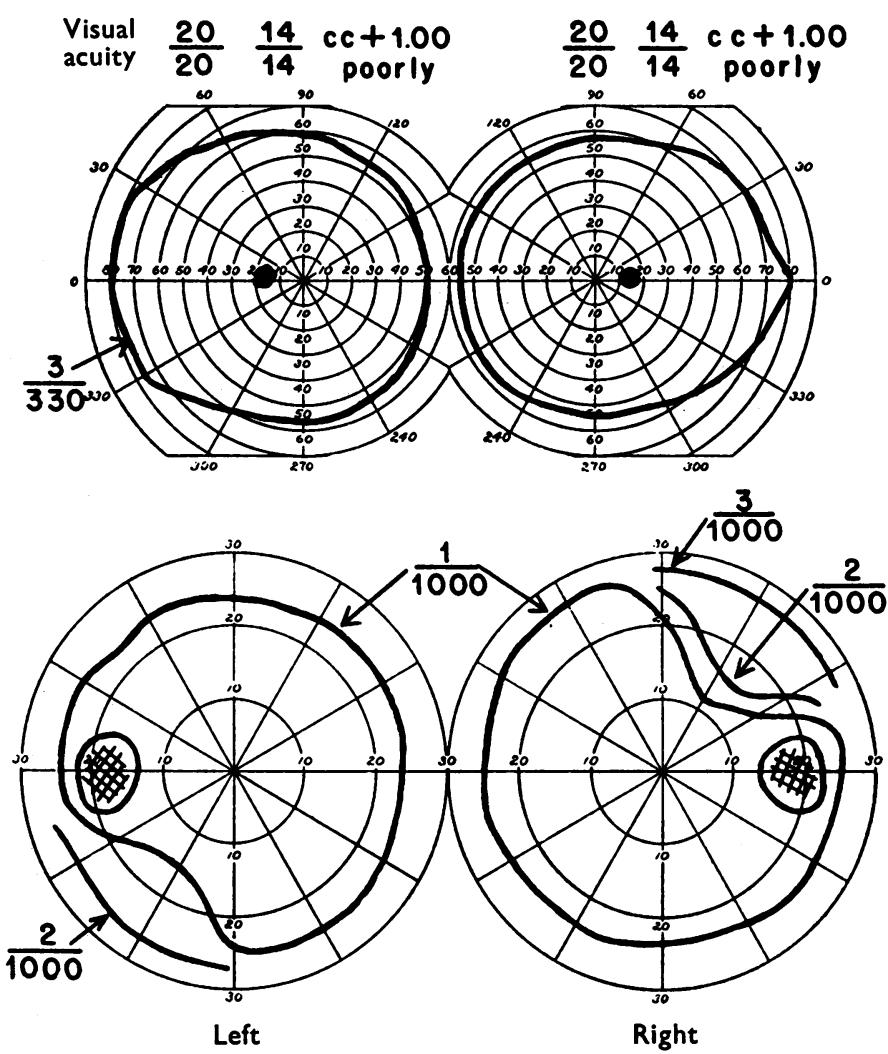

FIG. 16.-Visual fields plotted on July 26, 1956, in Case 5.

It was eventually discovered that chiasmal neuritis had been caused by chemicals containing benzene which the patient had used to spray a chicken coop.

It was felt that early surgical exploration was indicated because of the rapid and progressive visual loss sustained by this patient when no obvious cause could be found after careful clinical investigation. At a later date it was possible to determine that a chemical agent was most probably responsible for the condition.

\section{Summary and Conclusions}

An analysis of some of the clinical entities that produce acute visual failure illustrates the need for prompt neurosurgical intervention to prevent irreparable damage to the visual system. The need for a complete and accurate medical, neurological, and ophthalmological investigation in any of these conditions is apparent, and every effort should be made to arrive at the correct diagnosis early, so that definitive treatment can be instituted promptly. A 

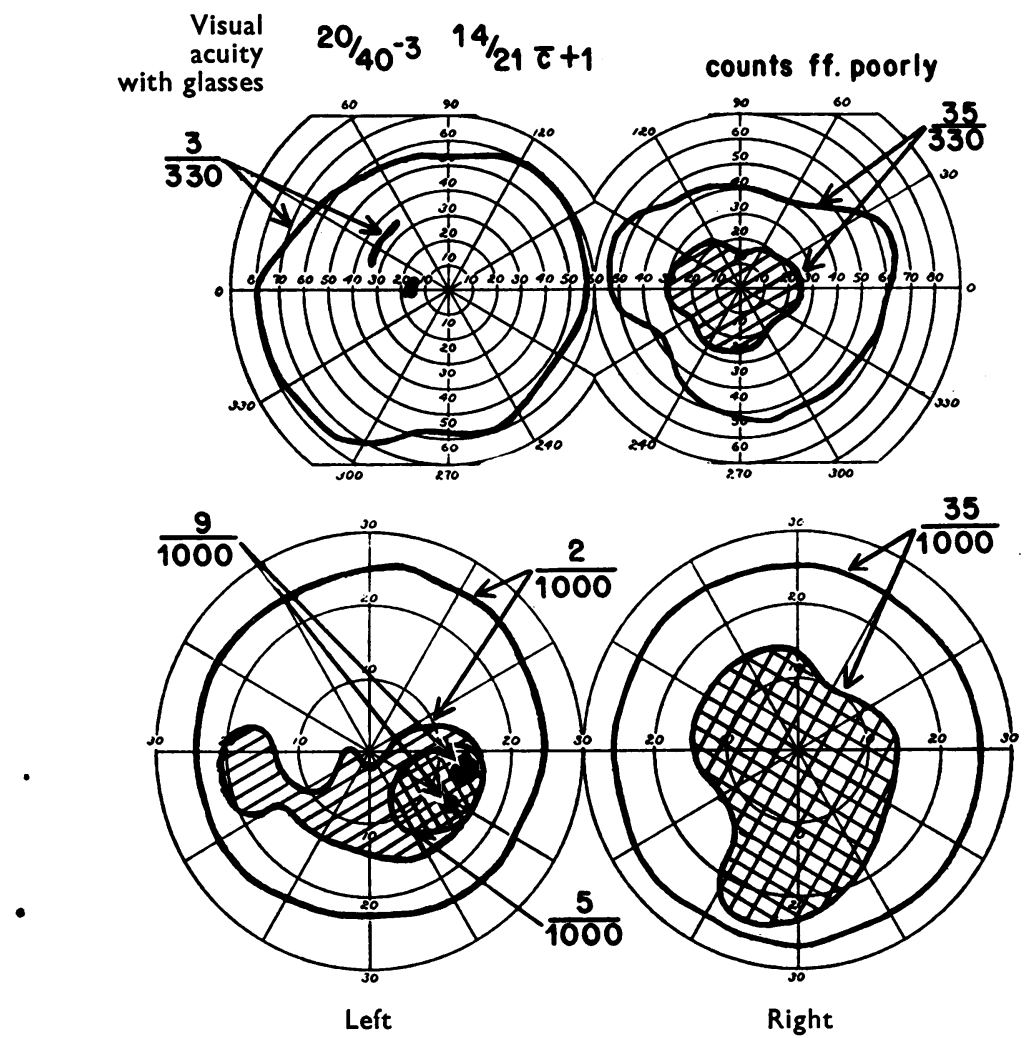

Fig. 17.-Visual fields plotted on December 15, 1956, in Case 5.

diagnosis of retrobulbar neuritis or multiple sclerosis is not uncommon in a hasty evaluation of rapid decrease in visual acuity. This mistake may cause irreparable damage to the optic nerves before the correct diagnosis is made, and surgical treatment may prove discouraging because it is carried out too late.

Five reports of cases are presented to illustrate the difficulties of diagnosis that confront the physician in the management of the problems described. The pitfalls of a hasty diagnosis are obvious, and in many instances definitive treatment can hope only to prevent additional visual loss, since the type of pathological process present of necessity prevents restoration of vision on the affected side. The fact that occasionally an apparently hopeless situation can be retrieved justifies careful search for lesions which can be treated successfully by surgical intervention.

\section{REFERENCES}

BAKER, G. S., and RUCKER, C. W. (1950). J. Neurosurg., 7, 377.

Hughes, B. (1954). "The Visual Fields: A Study of the Applications of Quantitative Perimetry to the Anatomy and Pathology of the Visual Pathways". Thomas, Springfield, Ill. 


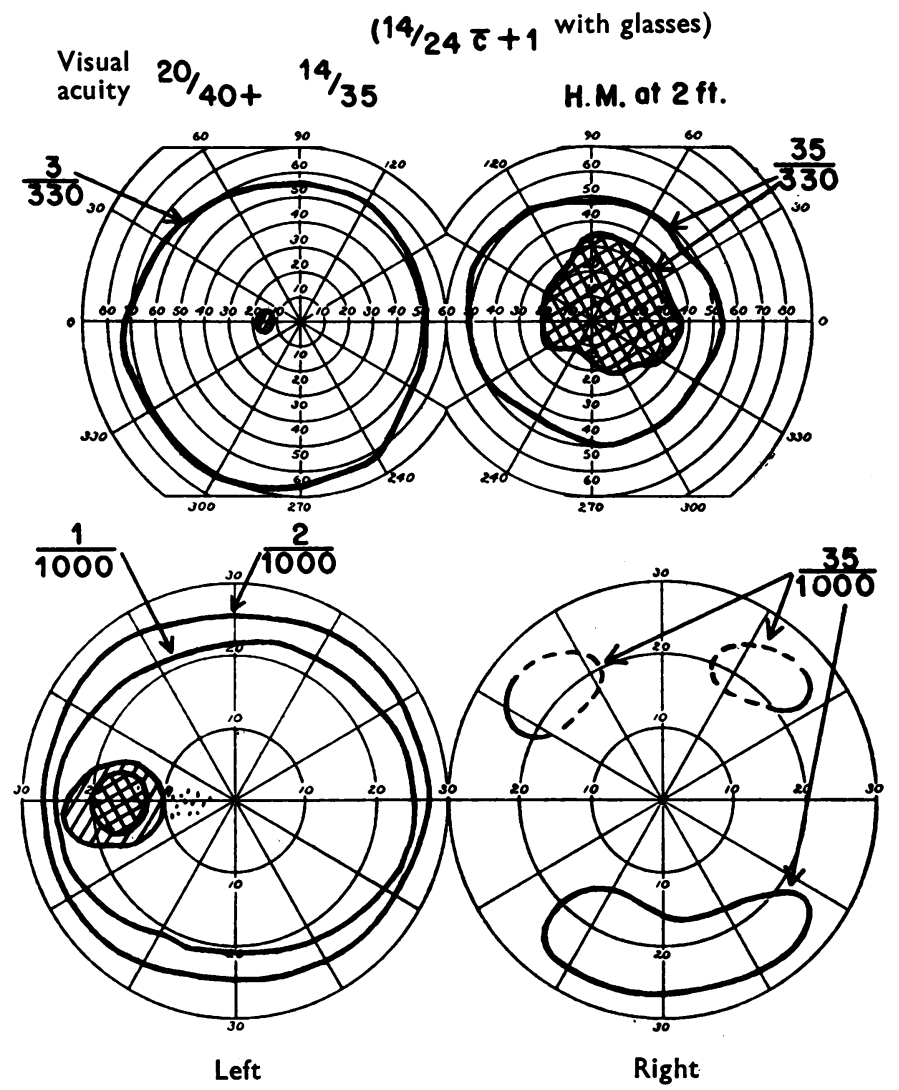

FIG. 18.-Visual fields plotted on January 5, 1957, in Case 5.

Jefrerson, G. (1955). "Further Concerning Compression of the Optic Pathways by Intracranial Aneurysms", in "Clinical Neurosurgery: Proceedings of the Congress of Neurological Surgeons", pp. 55-103. Williams and Wilkins, Baltimore.

List, C. F., Williams, J. R., and Balyeat, G. W. (1952). J. Neurosurg., 9, 177.

LOVE, J. G., DoDGE, H. W., Jr., and BAIR, H. L. (1955). A.M.A. Arch. Ophthal., 54, 386.

MacCarty, C. S., Lille, J. C., Daly, D. D., Hollenhorst, R. W., and Holman, C. B. (1957). Ibid., 58, 747.

Norlén, G. (1952). Proc. roy. Soc. Med., 45, 291.

UIHLEIN, A., BALFour, W. M., and Donovan, P. F. (1957). J. Neurosurg., 14, 140.

- and WeYAND, R. D. (1953). A.M.A. Arch. Ophthal., 49, 261. 